\title{
EFEITO DE DIFERENTES TÉCNICAS DE ENRIQUECIMENTO AMBIENTAL EM CATIVEIRO DE ONÇAS SUÇUARANAS (Puma concolor)
}

\section{EFFECT OF DIFFERENT ENVIRONMENTAL ENRICHMENT TECHNIQUES IN CAPTIVITY OF Puma concolor}

\author{
Gisele Dela Ricci ${ }^{*}$ \\ Cesar Henrique Branco ${ }^{2}$ \\ Rafael Teixeira Sousa ${ }^{1}$ \\ Cristiane Gonçalves Titto ${ }^{1}$ \\ ${ }^{1}$ Universidade de São Paulo, São Paulo, SP, Brasil. \\ ${ }^{2}$ Bosque e Zoológico de Ribeirão Preto, Ribeirão Preto, SP, Brasil. \\ “Autora para correspondência - giseledelaricci@usp.br
}

\begin{abstract}
Resumo
Objetivou-se avaliar os efeitos da implementação de diferentes técnicas de enriquecimento ambiental sobre a atividade comportamental de onças suçuaranas (Puma concolor) mantidas em cativeiro. Para este estudo, foram utilizadas cinco onças suçuaranas, quatro machos e uma fêmea, pertencentes ao Zoológico Municipal de Ribeirão Preto-SP. Para fins de enriquecimento ambiental, nos recintos dos felinos foram introduzidas bolas confeccionadas com cipó e sisal e, também, caixas de papelão com carne bovina ou erva cidreira e canela. Os dados de comportamento foram submetidos à análise descritiva. Mesmo em cativeiro, os animais apresentaram comportamento de demarcação territorial, como urinar e rugir em alguns momentos em que se sentiam ameaçados. Ademais, foi verificado um maior interesse das onças pelas caixas de papelão com carne bovina em detrimento daquelas que continham erva cidreira e canela. Foi observado também um entretenimento das onças com as bolas de cipó e sisal. Esses achados sugerem êxito nas estratégias de enriquecimento ambiental estudadas, o que foi evidenciado pelo interesse dos felinos nos diferentes materiais avaliados. Além disso, os enriquecimentos utilizados neste estudo são estratégias simples e de baixo custo que contribuem para o bem-estar de onças suçuaranas (Puma concolor), podendo ser aplicadas em outras instituições que abrigam esses animais.
\end{abstract}

Palavras-chave: ambiência; bem-estar; comportamento animal; recinto

\begin{abstract}
:
The objective of this study was to evaluate the effects of the implementation of different environmental enrichment techniques on the behavior of Puma concolor kept in captivity. Five Puma concolor, four males and one female, belonging to the Municipal Zoo of Ribeirão Preto-SP, were used for this study. For purposes of environmental enrichment, vine and sisal balls and cardboard boxes containing beef or lemon grass and cinnamon were introduced into the enclosures of the felines. The behavioral data were submitted to descriptive analysis. Even in captivity, the animals showed territorial demarcation behavior, such as urinating and roaring when feeling threatened. In addition, there was a greater interest of the Puma concolor for the cardboard boxes with beef to the detriment of those that contained lemon grass and cinnamon. The cougars also showed to be entertained by the
\end{abstract}


vine and sisal balls. These findings suggest success of the environmental enrichment strategies studied, which was evidenced by the interest of the felines in the different evaluated materials. In addition, the enrichment strategies used in this study represent simple and low-cost strategies that contribute to the well-being of Puma concolor and can be applied in other institutions that house these animals.

Keywords: ambience; animal behavior; enclosure; welfare.

Recebido em: 09 de julho de 2017.

Aceito em: 19 de janeiro de 2018.

\section{Introdução}

$\mathrm{Na}$ literatura especializada em animais selvagens, é possível encontrar com facilidade uma vasta gama de estudos sobre o impacto de fatores estressores no comportamento e bem-estar de animais mantidos em cativeiro ${ }^{(1-4)}$. Entretanto, existe uma escassez de artigos científicos e uma grande demanda de conhecimentos científicos voltados aos efeitos do enriquecimento ambiental no comportamento e na promoção de qualidade de vida em onças suçuaranas (Puma concolor) cativas. Dentro desse contexto, um melhor entendimento sobre o uso do enriquecimento ambiental, através de técnicas de entretenimento mediante o uso de brincadeiras e dificuldades propostas, pode favorecer adequações do ambiente criatório visando o bem-estar animal nessa espécie.

O Puma concolor é o segundo maior felino brasileiro ${ }^{(5)}$. É um predador generalista excepcionalmente bem-sucedido e sua resiliência provavelmente o ajudou a sobreviver à última extinção dos grandes felinos durante o Pleistoceno ${ }^{(3)}$. Assim como os felinos de modo geral, as onças suçuaranas são primariamente noturnas e crepusculares, com picos de atividade ao entardecer e ao amanhecer e com atividade diurna limitada ${ }^{(6)}$.

$\mathrm{Na}$ América Central e na América do Sul, a onça suçuarana ainda ocorre por toda parte de sua distribuição histórica; entretanto, os estudos focais têm sido feitos somente na América do Norte ${ }^{(3)}$. No Canadá, o Puma concolor tem sido extinto em boa parte de sua extensão inicial e essa espécie também foi essencialmente eliminada de grande parte do leste dos EUA dentro dos 200 anos seguintes à colonização europeia ${ }^{(7)}$. Nesse sentido, a manutenção do Puma concolor em zoológicos, visando sua conservação, é muito importante para a sobrevivência dessa espécie, que, em vida livre, como a maioria dos carnívoros, normalmente necessita de uma grande extensão territorial para sobreviver e esta diminui cada vez mais em função da intensificação do desmatamento, agricultura e pecuária ${ }^{(8)}$.

Dessa forma, o ambiente cativo tem se tornado uma importante ferramenta para a conservação e manutenção dessas espécies. Porém, o cativeiro impõe aos animais selvagens condições muito diferentes daquelas encontradas em seus ambientes naturais ${ }^{(1)}$. Sabe-se que comportamentos incomuns à espécie, tais como agressividade excessiva, estereotipias ou inatividade, são considerados resultados do cativeiro inadequado ${ }^{(9,10)}$. Por essa razão, é importante que os recintos tenham ambientes enriquecidos e a equipe de técnicos e tratadores conheça o comportamento dos animais para que felinos selvagens mantidos nessas condições apresentem um bom desenvolvimento físico e psicológico $^{(11)}$.

O bem-estar dos felinos tornou-se uma preocupação para profissionais que interagem direta e indiretamente com esses animais, uma vez que são animais frequentes em zoológicos ${ }^{(12)}$. $\mathrm{O}$ enriquecimento ambiental é considerado como procedimento para melhorar o bem-estar psicológico 
para felinos, primatas e outros mamíferos em cativeiro, incluindo animais de produção ${ }^{(9,13)}$.

Já o enriquecimento comportamental consiste na inserção de objetos que modifiquem o ambiente físico ou social dos animais, melhorando a qualidade de vida, proporcionando condições mais próximas das necessidades etológicas e tornando o ambiente mais rico ${ }^{(14)}$. O procedimento para a modificação do ambiente, a partir de enriquecimentos, é definido como uma série de medidas que melhoram o ambiente físico e social, com o uso de técnicas inovadoras e simples, capazes de proporcionar condições mais próximas das adequadas para o desempenho de suas necessidades etológicas ${ }^{(14)}$.

$\mathrm{O}$ ambiente enriquecido e a introdução de ferramentas que aprimorem o cativeiro são considerados como práticas essenciais para a redução de reações adversas ou agressivas e as mudanças na rotina diária são medidas suficientes para estimular e melhorar o psicológico, o físico e o bem-estar dos animais cativos $^{(14,15)}$. Dada a importância da avaliação do comportamento para a promoção do bemestar de animais selvagens mantidos em zoológicos, objetivou-se mensurar a qualidade ambiental do recinto e a etologia de onças suçuaranas (Puma concolor) a partir da introdução de enriquecimentos sensoriais e alimentícios.

\section{Material e Métodos}

O presente estudo foi realizado no Bosque e Zoológico Fábio Barreto localizado em uma área de mata atlântica, situada na cidade de Ribeirão Preto no Estado de São Paulo. A cidade possui clima tropical, com classificação Aw de Koppen-Geiger, com temperaturas entre 16,9 e 23,2 ${ }^{\circ} \mathrm{C}$ e uma pluviosidade de 1422,5 mm. Estando a uma altitude de cerca de 531 metros, apresenta duas estações do ano bem distintas, uma chuvosa, que se estende de outubro a março, e outra mais seca, que vai de abril a setembro $^{(16)}$. As observações e coletas de dados se estenderam no período de julho a agosto de 2011. No período, foram estudadas cinco onças suçuaranas (Puma concolor), sendo quatro machos e uma fêmea. O experimento foi aprovado pela Comissão de Ética no Uso de Animais (CEUA) do Bosque e Zoológico Fábio Barreto, protocolado sob o n.020/11.

Os recintos das suçuaranas estão situados junto ao setor dos grandes felinos do zoológico e é cercado por vegetação nativa. O recinto I tem um lago, com vegetação e dormitório para os animais com 300 $\mathrm{m}^{2}$ (Figura 1). Neste estão alojados dois animais, uma fêmea e um macho, com aproximadamente nove anos e que chegaram ainda filhotes ao zoológico. No recinto II estão alojados três animais machos de com aproximadamente nove anos, que chegaram ainda filhotes ao zoológico, com cobertura para ventilação e troncos para a distração dos animais com dimensão de $125 \mathrm{~m}^{2}$ (Figura 2).

Foram utilizados como forma de enriquecimento os seguintes materiais: caixas de papelão, carne bovina e cordões de sisal para o enriquecimento alimentar e canela em pó, erva doce para o enriquecimento sensorial. Optou-se por esses materiais por serem de fácil obtenção e versatilidade para montagem dos objetos.

O enriquecimento alimentício foi confeccionado utilizando-se caixas de papelão contendo carne bovina, distribuídas em diferentes regiões do recinto, porém distantes do enriquecimento sensorial. A canela em pó e a erva doce também foram aplicadas dentro de caixas de papelão de diferentes tamanhos e colocadas em vários locais do recinto com exceção dos lugares de alimentação e defecção dos animais. Foi utilizada uma bola de cipó amarrada com corda de sisal de tamanho médio no exterior do recinto, com a finalidade de observar a reação dos animais quanto à proteção e interesse. 


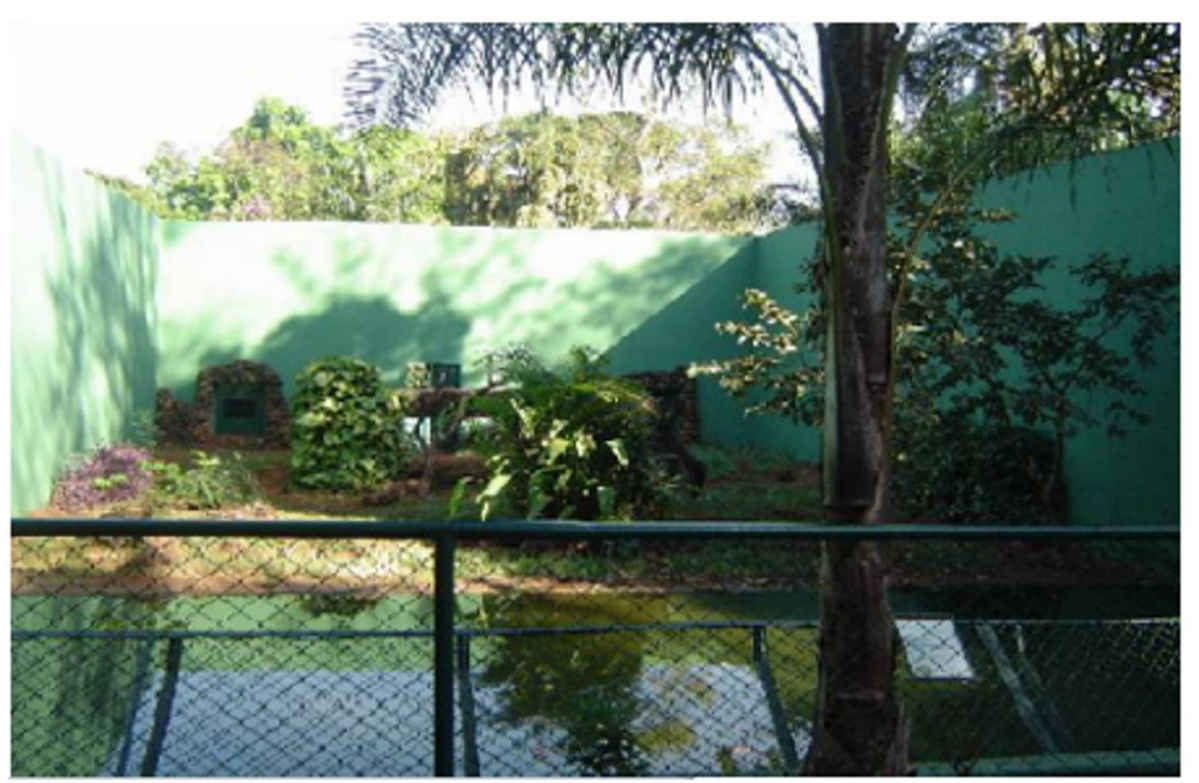

Figura 1: Foto do recinto I das onças-pardas localizado no Bosque e Zoológico Fábio Barreto, Ribeirão Preto-SP

Fonte: Gisele Dela Ricci

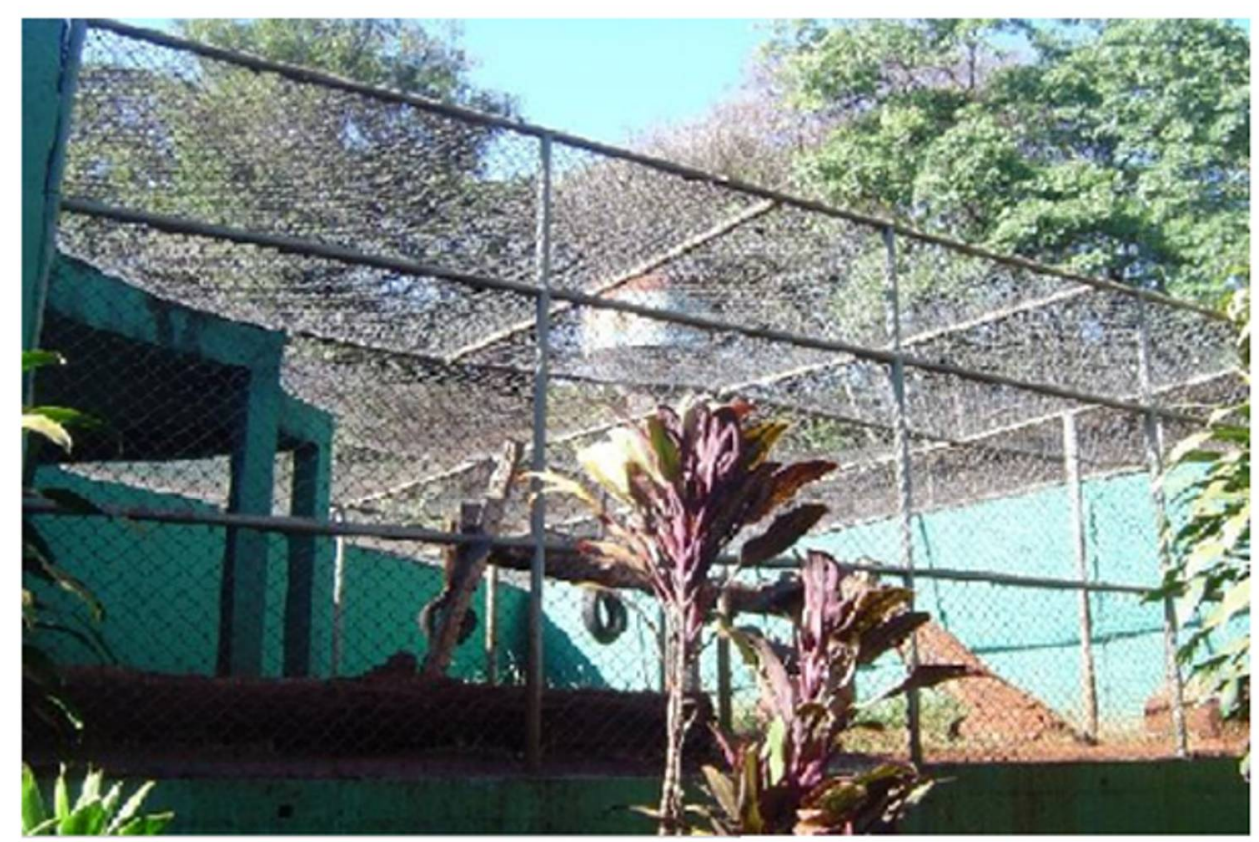

Figura 2: Recinto I das onças-suçuaranas localizado no Bosque e Zoológico Fábio Barreto, Ribeirão Preto-SP

Fonte: Gisele Dela Ricci

Para obtenção de dados comportamentais, foi construído um etograma já validado anteriormente para amostragem da frequência de realização de cada comportamento das suçuaranas ${ }^{(17)}$. $\mathrm{O}$ enriquecimento aromático foi inserido nas baias no momento da limpeza, quando os animais eram contidos na parte de trás dos cativeiros e a caixa/bola era inserida por meio de arremesso por tratadores treinados para essa função. A observação se deu na forma dirigida conforme ${ }^{(18)}$ de modo a 
não afetar o comportamento natural das mesmas. As observações das onças foram realizadas de dez em dez minutos, durante quatro horas, cinco vezes por semana durante um mês. Na identificação do comportamento, adotou-se etograma elaborado com base no comportamento das onças mantidas em cativeiros. Os observadores foram treinados para identificar os principais pontos comportamentais dos animais, baseando-se em escala previamente definida. Foram feitas observações iniciais no momento e posteriormente à introdução dos materiais para o enriquecimento ambiental. A Tabela 1 indica a descrição dos comportamentos avaliados durante a observação das suçuaranas.

Tabela 1. Definição dos comportamentos das onças suçuaranas (Puma concolor) avaliados durante o estudo

\begin{tabular}{|c|c|}
\hline Comportamento & Descrição \\
\hline Interesse & $\begin{array}{c}\text { Interesse ou obsessão pelo material inserido no cativeiro por } \\
\text { determinado sexo }\end{array}$ \\
\hline Ócio & $\begin{array}{l}\text { Ato em que o animal dorme, permanece deitado, sentado, em pé, } \\
\text { sem função aparente }\end{array}$ \\
\hline Agonistico & Agressividade entre os animais de um mesmo recinto \\
\hline Estereotipado & Apresentação de atividades compensatórias \\
\hline Exploratório & Ato de cheirar, fuçar ou cavar o ambiente \\
\hline Fuga & Ato de esconder-se do público \\
\hline Alimentação & Ato de comer ou beber \\
\hline
\end{tabular}

\section{Resultados e discussão}

Em cativeiro, as onças suçuaranas (Puma concolor) apresentaram atitudes de interesse e/ou curiosidade e obsessão ao longo do trabalho cotidiano de enriquecimento ambiental. Nos dados comportamentais das suçuaranas, foi evidenciado que os animais têm preferência a determinadas áreas do recinto. Entre elas estão os cantos sombreados, aqueles fechados com vegetação e os que estão longe das grades de frente para o público. A relação entre a presença de público nos zoológicos e o comportamento encontrado neste estudo para os felinos, caracterizando os visitantes como estressores em potencial, já foi verificada em outro estudo ${ }^{(19)}$.

Com frequência, suçuaranas machos e fêmea escondiam-se atrás de pedras e pequenos arbustos dispostos dentro do recinto. Mudanças de comportamento acontecem no momento da alimentação, em função da presença de público e geralmente pelo calor excessivo. Da mesma forma, Kerley et al. ${ }^{(20)}$ avaliaram tigres selvagens, demonstrando que estes felinos ingeriram maior quantidade de alimento e permaneceram mais tempo em locais que não tinham interferência humana.

Os carnívoros dedicam grande parte do seu tempo ao comportamento de caça em habitats ${ }^{(21)}$. Em cativeiro, as oportunidades de expressão desse comportamento são reduzidas, porém a motivação para ele continua forte ${ }^{(22)}$. Margulis et al. ${ }^{(2)}$ estudaram seis diferentes espécies de felinos (Panthera 
leo, Panthera pardus orientalis, Phantera tigres altaica, Phantera uncia, Neofelis nebulosa e o Prionailurus viverrinus) em ambientes cativos e verificaram que a presença dos visitantes não teve efeito em qualquer uma das espécies estudadas. Todavia, já foi reportado que visitantes afetam o comportamento de leopardos indianos (Panthera pardus) cativos $^{(23)}$. Um estudo de 12 diferentes espécies de primatas mostrou que o público ativo influenciou os primatas a realizar, significativamente, mais atividade locomotora e a interagir mais com esse público do que eles interagiam com um público passivo, com os maiores grupos tendo uma maior influência ${ }^{(4)}$.

A inserção de enriquecimentos sensoriais melhora a saúde física, emocional e espiritual dos animais, podendo ser utilizados produtos extraídos de vegetais, como a camomila, erva doce e óleos essenciais, responsáveis por reduzirem a ansiedade, estresse e dor em pessoas ${ }^{(29)}$. A aromaterapia melhora a capacidade do animal em investigar o ambiente, podendo marcar o território esfregando-se nos novos odores, permitindo estimulação do sentido olfativo dos animais ${ }^{(30)}$.

Neste estudo, os machos demonstraram maior interesse e disposição para abrir as caixas com carne e menos interesse pelo enriquecimento sensorial. Observou-se que a fêmea se esfregou nos locais estratégicos onde foram postos os enriquecimentos com erva doce e canela ficando mais atenta ao enriquecimento, mesmo nas horas mais quentes do dia. Além do mais, a fêmea mostrou-se mais curiosa e propensa a brincadeiras com animais da mesma espécie ou com suas presas. Dentre os quatro machos, três tiveram interesse pelo alimento de dentro da caixa e um quarto animal mostrou obsessão pelo material, ou seja, conseguir abrir e se alimentar (Tabela 2). Van Dyke et al. ${ }^{(6)}$ indicaram que, assim como os felinos de modo geral, as onças suçuaranas são primariamente noturnas, com picos de atividade ao entardecer e ao amanhecer e com atividade diurna limitada, conceito que contrapõe o comportamento encontrado neste estudo. Entre as possíveis explicações, podemos indicar a falta rotineira dos enriquecimentos, a fase fisiológica dos animais, a presença do público, entre outros fatores.

A escolha do material e do tipo dos objetos que são inseridos nos cativeiros para melhorar o grau de entretenimento dos animais precisa atender requisitos importantes, como comportamentos típicos das espécies, habilidades dos animais, entre outros, uma vez que a má escolha pode limitar a funcionalidade do enriquecimento ambiental. Testes de preferência são realizados buscando estabelecer a capacidade e eficiência do enriquecimento para a espécie específica a ser beneficiada perante os diferentes tipos de enriquecimento disponíveis ${ }^{(24-26)}$.

No zoológico em estudo, os recintos possuíam enriquecimentos fixos, como pneus, dispostos em diferentes locais. Também, eram fornecidos enriquecimentos duas vezes por semana para as suçuaranas. No entanto, as onças apresentavam estresse constante, aparentado a partir de estereotipias e agressividade. Notou-se que os animais perderam o interesse pelo enriquecimento fixo (pneus) (Figura 3) e que o enriquecimento diário e diferente apresentou maior variabilidade de comportamento (Figura 4). A eficiência de qualquer enriquecimento está ligada à sua capacidade de motivação do comportamento típico de uma espécie específica ${ }^{(27)}$.

O estudo do comportamento de animais em cativeiros permite compreender como condições restritas de ambiente modificam o comportamento típico dos animais e observar quais são as melhores alternativas encontradas para a adaptação à vida em recintos ${ }^{(28)}$. A incapacidade de realizar atividades naturais em cativeiro causa estresse nos animais ${ }^{(1)}$ com o aparecimento de comportamentos atípicos como as estereotipias, inatividade ou a agressividade fora do padrão para a espécie, todos definidos como resultado do estresse pelo cativeiro não enriquecido ${ }^{(9,10)}$. Neste estudo, durante a coleta dos dados, não foram observados nenhum tipo de comportamento agressivo entre os animais. No entanto, 
isso pode não representar boa qualidade de vida e bem-estar animal adequado e sim boa adaptabilidade dos animais aos próprios animais, uma vez que foram criados juntos, desde filhotes, no mesmo recinto e com as mesmas expectativas.

É primordial que os ambientes sejam enriquecidos e que o grupo de funcionários, incluindo corpo técnico, tratadores e afins conheçam o comportamento dos animais, para que as espécies sejam mantidas em condições adequadas para o desempenho de atividades típicas, buscando desenvolver o bem-estar físico e psicológico dos animais em cativeiro ${ }^{(11)}$.

Os achados neste e em outros estudos indicam a necessidade da realização de mais análises e observações comportamentais para a quantificação da interferência gerada pelo ambiente criatório de zoológicos sobre as onças suçuaranas que lá se encontram e, também, para avaliar outras variáveis que também podem estar interferindo no comportamento dos animais cativos.

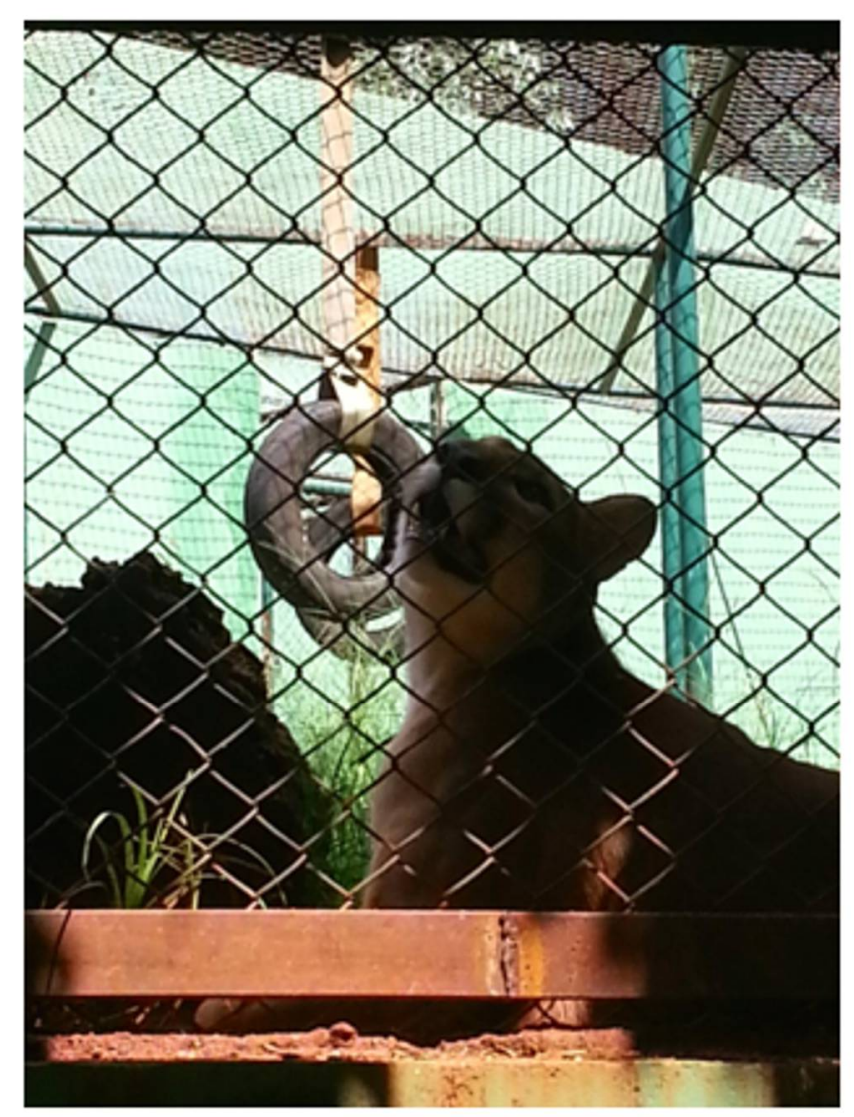

Figura 3. Detalhe do Puma concolor estressado, apesar da presença de enriquecimento ambiental fixo no Bosque e Zoológico Fábio Barreto, Ribeirão PretoSP

Fonte: Gisele Dela Ricci 


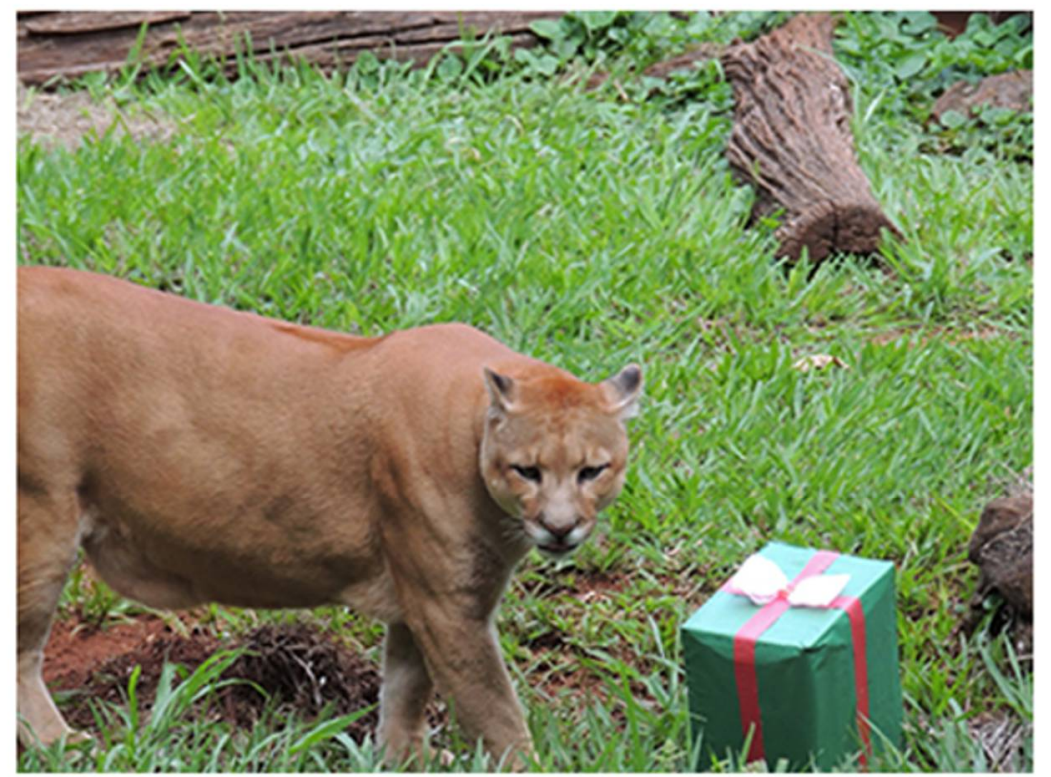

Figura 4. Detalhe da interação entre o Puma concolor e caixa de papelão utilizadas no enriquecimento ambiental no Bosque e Zoológico Fábio Barreto, Ribeirão Preto-SP Fonte: Bosque e Zoológico Fábio Barreto

Tabela 2. Resultados do comportamento observado em suçuaranas em recintos com enriquecimentos ambientais alimentares e sensoriais

\begin{tabular}{cccc}
\hline Enriquecimento & Material & Macho & Fêmea \\
\hline Alimentar & Caixas de papelão & I/IA/O & PI/ A \\
Alimentar & Bola de cipó & I & I \\
Sensorial & Erva doce & I & I \\
Sensorial & Canela em pó & PI & I \\
\hline
\end{tabular}

I: Interesse/ curiosidade; IA: Interesse somente no alimento; O: Obsessão pelo material; N: Neutro; PI: Pouco interesse

\section{Conclusão}

Os achados nesta pesquisa sugerem êxito nas estratégias de enriquecimento ambiental estudadas, o que foi evidenciado pelo interesse dos felinos nos diferentes materiais avaliados. Tanto as caixas contendo carne bovina ou erva doce e canela em pó quanto as bolas de cipó e sisal mostraram-se efetivas na estimulação da atividade de Puma concolor mantidos em cativeiro. Além disso, os 
enriquecimentos utilizados neste estudo são estratégias simples e de baixo custo que contribuem para o bem-estar de onças suçuaranas (Puma concolor) cativas, podendo ser aplicadas em outras instituições que abrigam esses animais.

\section{Referências}

1 Sellinger RL, Ha JC. The effects of visitor density and intensity on the behavior of two captive jaguars (Panthera onca). Journal of Applied Animal Welfare Science, 2005; 8(4):233-244. Disponível em: http://www.tandfonline.com/doi/abs/10.1207/s15327604jaws0804_1 Inglês

2 Margulis SW, Hoyos C, Anderson M. Effects of felid activity on zoo visitor interest. Zoo Biology,2003; 22: 587-599. Disponível em: http://onlinelibrary.wiley.com/doi/10.1002/zoo.10115/full Inglês

3 Nowell K, Jackson P. Wild cats. Status Survey and Conservation Action Plan. IUCN/SSC Cat Specialist Group, Gland, Switzerland; 1996. Inglês

4 Hosey GR, Druck PL. The influence of zoo visitors on the behavior of captive primates. Applied Animal Behaviour $\quad$ Science, 1987 ; 18: 19-29. Disponível em: http://www.sciencedirect.com/science/article/pii/0168159187902516 Inglês

5 Margarido TCM, Braga FG. Mamíferos. In: Mikich SB, B'ernils RS. (Eds.). Livro vermelho da fauna ameaçada no estado do Paraná. Curitiba: Secretaria Estadual de Meio Ambiente, Instituto Ambiental do Paraná; 2004 p.25 -142. Português

6 Van Dyke FG, Brocke RH, Shaw HG, Ackerman BB, Hemker TP, Lindzey FG. Reactions of mountain lions to logging and human activity. The Journal of Wildlife Management, 1986; 50(1): 95-102. Disponível em: https://www.jstor.org/stable/3801496?seq=1\#page scan tab contents Inglês

7 Wright BS. The ghost of North America - the story of the eastern panther. Vantage, New York; 1959. Inglês

8 Lindzey FG. Mountain lion. In: Novak M, Baker J, Obbard M, Malloch B (Eds.) Wild furbearer management and conservation in North America. Ontario Ministry of Natural Resources, Toronto; 1987.p.656-668. Inglês

9 Carlstead K. Effects of captivity on the behavior of wild mammals In: Kleiman D, Allen M, Thompson M, Lumpkin S. (Eds) Wild Mammals in Captivity. Chicago, University of Chicago Press; 1996. p.317-333. Inglês

10 Morato RG, Conforti VA, Azevedo FC, Jacomo ATA, Silveira L, Sana D, Nunes ALV, Guimarães M A B $\mathrm{V}$, Barnabe R C. Comparative analyses of semen and endocrine characteristics of free-living versus captive jaguars (Panthera onca). Reproduction, 2001; 122: 745-751. Disponível em: http://www.reproductiononline.org/content/122/5/745.short Inglês

11 Campos B, Queiroz VS, Morato RG, Genaro G. Padrão de atividade de onças pintadas (Panthera onca Linnaeus, 1758) mantidas em cativeiro-manejo e comportamento. Revista de Etologia, 2005; 7(2):75-77. Disponível em: http://pepsic.bvsalud.org/pdf/reto/v7n2/v7n2a04.pdf Português

12 Lossa G. Are wild animals suited to a travelling circus life? Animal Welfare, 2009; 18: 129-140. Disponível em:

http://www.dzivniekubriviba.lv/sites/default/files/are_wild_animals_suited_to_a_travelling_circus_life.pdf Inglês

13 Markowitz H. Behavioural enrichment in the Zoo. New York, Van Nostrand Rein- hold; 1982. 210p. Inglês

14 Boere V. Environmental enrichment for Neotropical primates in captivity: a review. Ciência Rural, 2001; 31:451-460. Disponível em: http://www.scielo.br/pdf/cr/v31n3/a31v31n3.pdf Inglês

15 Coe JC. Design and perception: making the zoo world real. Zoo Biology, 1985:197-208. Disponível em: file://C:/Users/Windows\%208.1/Downloads/Design\%20and\%20Perception\%201985\%20L.pdf Inglês 
16 CEPAGRI. Clima dos Municípios Paulistas. Disponível em: http://www.cpa.unicamp.br/outrasinformacoes/clima_muni_490.html/. Acesso: 09/06/2017.

17 Del-Claro K. Comportamento Animal-Uma introdução à ecologia comportamental. Jundiaí: Ed. Livraria Conceito; 2004.132 p. Português

18 Ferreira RA. Maior produção com melhor ambiente para aves, suínos e bovinos. Viçosa: Aprenda Fácil; 2005. $374 \mathrm{p}$.

19 Chamove A, Hosey G, Schaetzel P. Visitors excite primates in zoos. Zoo Biology, 1988; 7: 359-369. Disponível em: http://onlinelibrary.wiley.com/doi/10.1002/zoo.1430070407/pdf Inglês

20 Kerley LL, Goodrich JM, Miquelle DG, Smirnov EN, Quigley HB, Hornocker MG. Effects of roads and human disturbance on amur tigers. Conserv Biol, 2002; 16: 97-108. Disponível em: http://onlinelibrary.wiley.com/doi/10.1046/j.1523-1739.2002.99290.x/pdf Inglês

21 Shepherdson DJ, Carlstead K, Mellen JD, Seidensticker J. The influence of food presentation on the behavior of small cats in confined environments. Zoo Biology, 1993; 12: 203-216. Disponível em: http://onlinelibrary.wiley.com/doi/10.1002/zoo.1430120206/full Inglês

22 Mason G. Stereotypies: a critical review. Animal Behaviour, 1991; 41: 1015-1037. Disponível em: http://www.sciencedirect.com/science/article/pii/S0003347205806402 Inglês

23 Mallapur A, Chellam R. Environmental influences on stereotypy and the activity budget of Indian leopards (Panthera pardus) in four zoos in southern India. Zoo Biology, 2002; 21: 585-595. Disponível em: http://onlinelibrary.wiley.com/doi/10.1002/zoo.10063/pdf Inglês

24 Beattie VE, Walker N, Sneddon IA. Preference testing of substrates by growing pigs. Animal Welfare, 1998 $7(1): 27-34$. Disponível em: http:/www.ingentaconnect.com/content/ufaw/aw/1998/00000007/00000001/art00003 Inglês

25 Bench CJ, Rioja-Lang SM, Hayne SM, Gonyou HW. Group gestation sow housing with individual feedingII: How space allowance group size and composition, and flooring affect sow welfare, 2013;152(2-3): 218227. Disponível em: http://www.sciencedirect.com/science/article/pii/S1871141313000279 Inglês

26 Deboer SP, Garner JP, Lay DC, Eicher SD, Lucas JR, Marchant-Forde JN. Does the presence of a human affect the preference of enrichment items in young, isolated pigs? Applied Animal Behaviour Science, 2013; 143(2-4): 96-103. Disponível em: http://www.sciencedirect.com/science/article/pii/S0168159112003024 Inglês

27 Day JEL, Van de Weerd HA, Gonyou HW. The effect of varying lengths of straw bedding on the behaviour of growing pigs. Applied Animal Behaviour Science, 2008; 109 (2-4): 249-260. Disponível em: http://www.sciencedirect.com/science/article/pii/S0168159107000640 Inglês

28 Saad CEP, Saad FMOB, França J. Bem-estar em animais de zoológicos. Revista Brasileira de Zootecnia, 2011; 40:38-43. Disponível em: file:///C:/Users/Windows\%208.1/Downloads/BemEstar\%20em\%20Animais\%20de\%20Zool\%C3\%B3gicos.pdf Português

29 Fayazi S, Babashahi M, Rezaei M. The effect of inhalation aromatherapy on anxiety level of the patients inpreoperative period. Iranian Journal of Nursing and Midwifery Research, 2011; 16:278-283. Disponível em: https://zootecnia.jatai.ufg.br/up/186/o/TCC Thalita_Nattiele de Oliveira_Barbosa.pdf Português

30 Grams K. Suggested Guidelines for Carnivore Enrichment. Toronto Zoo. 2004. Disponível em: http://www.torontozoo.com/meet animals/enrichment/carnivore enrichment.htm Português 